\title{
Changes in intraocular pressure during and after the dark-room test
}

\author{
J. GLOSTER AND D. POINOOSAWMY \\ Institute of Ophthalmology, University of London
}

The dark-room test has an established place in the investigation of glaucoma, its purpose $\frac{0}{0}$. being to decide whether there is a threat of angle closure. The conduct of the test is simple of and is usually as follows. The intraocular pressures are measured and the patient is put in complete darkness for I hour, after which the intraocular pressures are measured again. The commonest indications for carrying out a dark-room test are a history suggestive of intermittent closed-angle glaucoma or the gonioscopic finding of narrowness of the angle $\stackrel{\circ}{工}$ or of peripheral anterior synechiae. The mechanism of the dark-room test is usually $\vec{z}$ assumed to be that the pupil dilates in the dark thereby causing the root of the iris to hinder aqueous outflow from a narrow angle.

Published work on the dark-room test up to 1957 was reviewed by Leydhecker (1960) and $\vec{\varphi}$ more recent papers have added little of significance; in the majority of these investigations $\omega$ the intraocular pressure was measured with the Schiøtz tonometer, using as little illumination as possible for the tonometer reading taken immediately after the patient had been in darkness for an hour. Under such conditions the errors of Schiøtz tonometry could account for a substantial part of the $8 \mathrm{~mm}$. $\mathrm{Hg}$ rise in pressure which is considered to $\frac{\mathscr{Q}}{\mathbb{Q}}$ indicate a positive result (Gloster, I 966 ). The errors of Goldmann applanation tonometry are lower, but in this method a moderate amount of light falls upon the eye, and it might $\frac{9}{3}$ be asked if this would reduce the intraocular pressure and so give a falsely low result in the dark-room test. Clearly this possibility depends upon the rate at which the intraocular pressure falls in an eye which is illuminated after the dark-room test, but this aspect of the test appears to have attracted relatively little interest. The original purpose of the present 0 investigation was to discover how fast the intraocular pressure fell when the patient was exposed to light again after the test, but the results also yielded some other facts about the dark-room test which are of general interest.

\section{Methods}

The tests were conducted on patients attending the Glaucoma Clinic at the Institute of Ophthalmology, London, and formed part of the routine clinical assessment of these patients, some of whom $\tilde{O}$ were known to have glaucoma and had been treated medically or surgically, some of whom were regarded as free from any sign of glaucoma as a result of the tests, and some of whom were being kept under observation as glaucoma suspects. Some patients who were using miotics were taken offo drops for 24 to 48 hours before the tests, while others continued to use their drops; continuation of treatment or otherwise was determined by the clinical requirements of the individual patient.? The intraocular pressures were measured with the Goldmann applanation tonometer using the $\frac{T}{0}$ combined drops of novesine and fluorescein described by Fenton (1965). The patient sat in $\mathrm{a} \underset{\mathbb{\Phi}}{\mathrm{P}}$ completely darkened room and wore red goggles to ensure that darkness was not interrupted by $\frac{\rho}{\mathbb{D}}$ the inadvertent opening of the door of the room. Two procedures were followed. 
(I) FIRST PROCEDURE (I 44 EYES)

The intraocular pressure was measured and the patient was put in darkness for I hour. Using as little light as possible, novesine-fluorescein drops were then instilled and intraocular pressures were quickly measured. The room lights were then put on (at an ordinary level of interior illumination) and further measurements of intraocular pressure were made at frequent intervals for the first 10 minutes, and then at $\mathrm{I} 5$ and 20 minutes after putting the room lights on. Further instillations of novesine-fluorescein drops were made as required for applanation tonometry.

(2) SEGOND PROCEDURE ( 72 EYES)

The intraocular pressure was measured; the patient remained in darkness for $I$ hour, after which the pressure was measured again as in the first procedure. The patient remained in darkness for a further 5 minutes when another measurement of intraocular pressure was made. The room lights were then put on; 5 minutes later another measurement of pressure was made and after a further 5 minutes in the light the final tonometric reading was taken. Usually, two or three instillations of novesine-fluorescein drops sufficed for the four tonometric readings taken after the patient had been in the dark. For each measurement of intraocular pressure, three readings were taken with the Goldmann tonometer.

MYDRIATIC TEST

In 39 eyes, a mydriatic test was also performed. A measurement was taken with the applanation tonometer, after which I per cent. mydrilate was instilled once or twice according to the pupillary response. Tonometry was repeated with the pupil semidilated and fully dilated. The result recorded was the maximum increase in pressure observed during dilatation.

\section{Results}

These are presented in the following order:

(A) The rise in intraocular pressure which occurred when the patient was in darkness for one hour. The two procedures did not differ in this respect and their results have therefore been combined.

(B) The fall in intra-ocular pressure after the patient had been in darkness:

(i) in the first procedure,

(ii) in the second procedure.

\section{(A) The rise in intraocular pressure in darkness}

The results in all 176 eyes are shown in the upper half of Fig. I, which shows that there were no eyes in which the pressure fell, and only five in which the pressure remained unchanged. A large proportion of eyes showed rises of 2 to $6 \mathrm{~mm}$. $\mathrm{Hg}$, with the peak frequency at $4 \mathrm{~mm}$. $\mathrm{Hg}$, and the largest rise was $\mathrm{I} 7 \mathrm{~mm}$. $\mathrm{Hg}$. The distribution of the rise in pressure was not Gaussian but skewed to the right; this was verified by plotting the results on a cumulative frequency graph. In fact, one could scarcely expect these results to show a Gaussian distribution since there were several populations of eyes, i.e. glaucomatous and non-glaucomatous, treated and untreated, and operated and non operated. In addition one has to bear in mind the possibility that not all the rise in pressure was due to darkness; part of the rise could have resulted from a spontaneous variation in intraocular pressure, and, in the case of glaucomatous eyes on medical treatment, part of the rise could have been due to the effect of the drops wearing off with the passage of time. In order to assess these possibilities, data were analysed from 176 eyes in which the pressures had been recorded at hourly intervals over the same time of the day as that in which the dark-room tests were done (almost all were done between 9.30 a.m. and 12 noon); these results were obtained from patients attending the glaucoma clinic for repeated tonometry ("phasing") as part of 
their routine investigation, and they are shown in the lower half of Fig. I. From the latte it can be seen that there was a general tendency for the intraocular pressure to remain almo the same while the dark-room tests were done, although there was a wide variation, from fall of $4 \mathrm{~mm}$. $\mathrm{Hg}$ to a rise of $6 \mathrm{~mm}$. $\mathrm{Hg}$; these changes in pressure showed an approximatef Gaussian distribution.

(I)

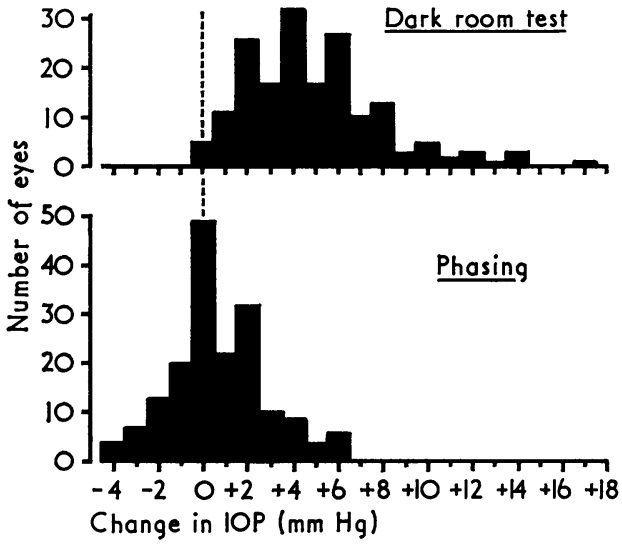

(2)

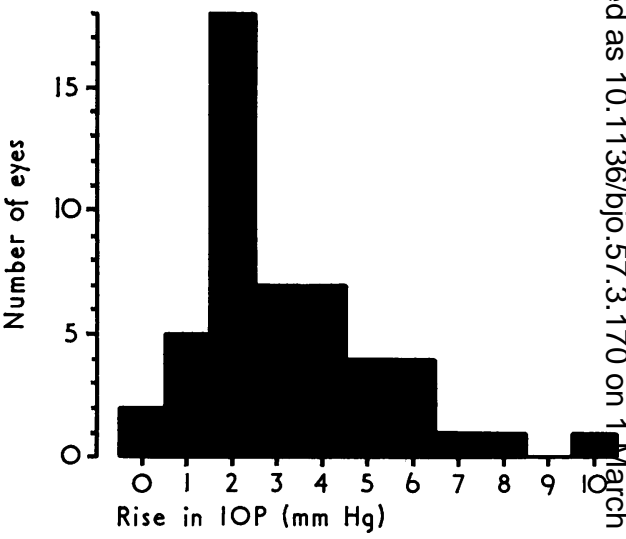

FIG. I Upper half shows the changes in pressure in 176 eyes during 1 hour in darkness. Lower half shorges changes in pressure in 176 eyes during 1 hour spent in the light

FIG. 2 Changes in pressure in fifty non-glaucomatous eyes during I hour in darkness

It has already been pointed out that the results shown in the upper half of Fig. I are frow a heterogeneous collection of eyes. It would be expected that a more homogeneous grou would be formed by those eyes which were regarded as non-glaucomatous at the end of the investigations. There were fifty such eyes and the distribution of the rise in pressure in darkness in this group is shown in Fig. 2. Again the distribution of the rises in pressure not Gaussian and there is a skew to the right; there is, however, no clear indication from Fig. 2 that we are dealing with more than a single population of responses of the intraoculap. pressure. The most homogeneous group consisted of thirty eyes with narrow angles which after investigation, were regarded as glaucomatous and which, at the time of testing, had. not been operated upon and were not on medical treatment; this group also showed distribution of responses very similar to that shown by the less homogeneous groups mentioned above.

The next question considered was whether there was any correlation between the rise im intraocular pressure in darkness and the initial level of pressure in the eye when the test began. An analysis was made of the results from 120 eyes which were not on medica9 treatment at the time of testing and had not been treated surgically. It was found tha隹 there was a significant positive correlation between the rise in pressure and the pressure if the eye at the beginning of the test $(r=0.26 ;$ d.f. $=1 \mathrm{I} 8 ; \mathrm{t}=2.93 ; 0.0 \mathrm{I}>\mathrm{P}>0)$. These eyes were then grouped according to their initial pressures. The average rises for each group are given in Table I, which indicates approximately the degree of dependence of the rise in pressure upon the initial pressure.

The next point to be examined was whether the amount by which the pressure rose wa? related to the configuration of the angle of the anterior chamber. The mean rise in prese sure in fourteen non-glaucomatous eyes with narrow angles was $3.93 \mathrm{~mm}$. $\mathrm{Hg}$ as compare 
Table I Dependence of rise in pressure on initial pressure

\begin{tabular}{lll}
\hline $\begin{array}{l}\text { Range of initial pressures } \\
(\mathrm{mm} . \mathrm{Hg})\end{array}$ & No. of eyes & $\begin{array}{l}\text { Mean rise in pressure } \\
(\mathrm{mm} . \mathrm{Hg})\end{array}$ \\
\cline { 1 - 1 } $10 \cdot 1-15 \cdot 0$ & 18 & $3 \cdot 4$ \\
$15 \cdot 1-20 \cdot 0$ & 57 & $4 \cdot 3$ \\
$20 \cdot 1-25 \cdot 0$ & 30 & $5 \cdot 4$ \\
$25 \cdot 1-30 \cdot 0$ & 10 & $6 \cdot 1$ \\
\hline
\end{tabular}

with $3.00 \mathrm{~mm}$. $\mathrm{Hg}$ in fifteen non-glaucomatous eyes with wide angles, but this difference was not significant. The use of results from non-glaucomatous eyes only to examine this relationship is perhaps erroneous because it has to be remembered that the dark-room tests reported here formed part of the routine examination of patients and therefore the result of the test had some bearing upon whether the patient was ultimately regarded as glaucomatous or non-glaucomatous. It is probably better therefore to combine the results of non-glaucomatous and glaucomatous eyes, provided that the latter have not been treated surgically and were not on medical treatment at the time of testing. When this comparison was made it was found that the mean rise in pressure in 44 eyes with narrow angles was $5.50 \mathrm{~mm}$. $\mathrm{Hg}$, while the rise in 43 eyes with medium or wide angles was $3.8 \mathrm{I} \mathrm{mm} . \mathrm{Hg}$, and this difference was significant. The mean initial pressure in the narrow-angled eyes was $19.45 \mathrm{~mm}$. $\mathrm{Hg}$ and that in the wide-angled eyes $18.70 \mathrm{~mm}$. $\mathrm{Hg}$; this difference was not significant, and therefore one cannot attribute the greater rise in pressure in the eyes with narrow angles to a higher initial pressure in these eyes as compared with those with medium and narrow angles.

The possibility arises therefore that the dependence of the rise in intraocular pressure in darkness upon the initial pressure, as shown in Table I, was due to the fact that the eyes with higher initial tensions tended to be those which had narrow angles while those with lower tensions tended to have wide angles. The dependence of the rise in intra-ocular pressure upon the initial level was therefore analysed for groups of eyes which were homogeneous as regards the type of angle; only unoperated eyes not on medical treatment were included. The results are given in Table II, which shows that there are highly significant correlations between the rise in pressure and the initial pressure in eyes having wide to medium angles, and a similar but less significant correlation in eyes having medium to

Table II Dependence of rise in intraocular pressure upon initial intraocular pressure for eyes with various types of angle

\begin{tabular}{|c|c|c|c|c|c|c|}
\hline \multirow{2}{*}{$\begin{array}{l}\text { Type of } \\
\text { angle }\end{array}$} & \multirow{2}{*}{$\begin{array}{l}\text { No. of } \\
\text { eyes }\end{array}$} & \multirow{2}{*}{$\begin{array}{l}\text { Coefficient of } \\
\text { correlation } r\end{array}$} & \multirow{2}{*}{$t$} & \multirow{2}{*}{ Significance } & \multicolumn{2}{|c|}{$\begin{array}{l}\text { Average intraocular } \\
\text { pressure (mm. } \mathrm{Hg})\end{array}$} \\
\hline & & & & & Initial & Rise \\
\hline Narrow & 44 & 0.09 & 0.58 & $\begin{array}{l}\text { Not significant } \\
\mathrm{P}>0.5\end{array}$ & $19 \cdot 45$ & $5 \cdot 50$ \\
\hline $\begin{array}{l}\text { Medium- } \\
\text { narrow }\end{array}$ & 33 & $0 \cdot 37$ & $2 \cdot 22$ & $\begin{array}{l}\text { Significant } \\
0.05>P>0.02\end{array}$ & $20 \cdot 18$ & $4^{\circ} 4^{8}$ \\
\hline $\begin{array}{l}\text { Medium and } \\
\text { medium-wide }\end{array}$ & 15 & 0.85 & $5 \cdot 81$ & $\begin{array}{l}\text { Highly significant } \\
0.01>P>0\end{array}$ & 19.00 & $3 \cdot 67$ \\
\hline Wide & 28 & 0.53 & $3 \cdot 19$ & $\begin{array}{l}\text { Highly significant } \\
0.01>P>0\end{array}$ & $18 \cdot 54$ & $3 \cdot 89$ \\
\hline
\end{tabular}


narrow angles. In contrast to these findings, eyes with narrow angles showed no significant correlation between the rise in pressure and the initial level.

In eyes with narrow angles, neither treatment with miotics at the time of the test nor previous operation (peripheral iridectomy or drainage) caused any significant alteration in the rise in pressure in darkness. These results are summarized in Table III; the $t$-tests showed that there were no significant differences between the mean rises in pressure or between the mean initial pressures.

Table III Lack of effect of miotics or operation on rise in pressure in darkness in eyes with narrow angles

\begin{tabular}{|c|c|c|c|c|}
\hline \multirow{2}{*}{ Type of eye } & & \multirow{2}{*}{$\begin{array}{l}\text { No. of } \\
\text { eyes }\end{array}$} & \multicolumn{2}{|c|}{$\begin{array}{l}\text { Mean intraocular } \\
\text { pressure }(\mathrm{mm} . \mathrm{Hg})\end{array}$} \\
\hline & & & $\begin{array}{l}\text { Rise in } \\
\text { darkness }\end{array}$ & Initial \\
\hline \multirow[t]{2}{*}{ Unoperated } & Not on miotics & $3^{0}$ & $6 \cdot 23$ & $19 \cdot 93$ \\
\hline & On miotics & 17 & $6 \cdot 53$ & 19.53 \\
\hline Operated & Not on miotics & 13 & $6 \cdot 00$ & $17 \cdot 31$ \\
\hline
\end{tabular}

As pointed out above, tonometry was always performed on the right eye before the left eye and it was therefore of some interest to compare the rises in pressure in the two eyes. This comparison was made for thirty patients, who had not had an operation, who were not on medical treatment at the time of testing, who did not have narrow angles, and in whom both eyes were tested. The mean rise in pressure in right eyes was $3.93 \mathrm{~mm} . \mathrm{Hg}$ and the mean initial pressure was $19.03 \mathrm{~mm} . \mathrm{Hg}$; the mean rise in left eyes was $4.17 \mathrm{~mm} . \mathrm{Hg}$ with a mean initial level of $19.83 \mathrm{~mm}$. $\mathrm{Hg}$. Neither the rise nor the initial level were significantly different in right eyes as compared with the left eyes $(t=0.37, \mathrm{P}>0.5$ for the rise; $t=0.66, \mathrm{P}>0.5$ for the initial level).

Ten experiments were carried out in which subjects sat in the light wearing goggles of the same type as in the dark-room tests, except that the front had been cut off one half of the goggles to allow light and air to reach one eye, while the red front of the other half had been cut off and replaced by transparent material, joined on so as to be airtight and thus allowing light but not air to reach the other eye. The intention was to discover whether air-tight occlusion of the eye had any effect on intraocular pressure. The results showed that there was no such effect, only two eyes showing any change, there being a rise of I $\mathrm{mm} . \mathrm{Hg}$ in one occluded eye and a fall of $\mathrm{I} \mathrm{mm}$. $\mathrm{Hg}$ in the opposite eye.

The results from 39 eyes, in which both dark-room and mydriatic tests were done, did not reveal any significant correlation between the rise in pressure in darkness and that induced by mydriasis $(r=0.096 ; t=0.587 ; \mathrm{P}>0.5)$.

(B) The fall in intraocular pressure after I $h r$ in darkness

(i) FIRST PROGEDURE

Tonometry was done immediately after the patient had been in darkness for $\mathrm{I}$ hour. The room lights were then put on and further measurements of intraocular pressure were made at frequent intervals for 20 minutes. The course in time of the fall in pressure was approximately exponential, as can be seen from Fig. 3 in which the average pressures from 104 eyes have been plotted. The results from individual eyes were submitted to a computer analysis, and most fitted reasonably well the equation $P_{t}=\left(P_{t}-P_{f}\right) e^{-k t}$, where $P_{t}$ was the 
pressure at any interval of time, $t$, after the patient was again exposed to light, $P_{i}$ was the initial pressure after $\mathrm{I}$ hour in darkness (i.e. at the beginning of exposure to light) and $P_{f}$ was the final pressure. Fig. 3 shows that there was an average total fall in pressure $\left(P_{i}-P_{f}\right)$ of nearly $8 \mathrm{~mm} . \mathrm{Hg}$, that the average rate of fall was about $2.0 \mathrm{~mm} . \mathrm{Hg} /$ minute immediately after exposure to light and that a steady final pressure was reached after about 15 to 20 minutes. The mean rise in pressure in the 104 eyes in the first procedure was $5.19 \mathrm{~mm} . \mathrm{Hg}$, and it is apparent therefore that, on the average, the final pressure reached at the end of the test was about $3 \mathrm{~mm}$. Hg lower than the pressure before the patient entered the dark room.

(ii) SECOND PROCEDURE

This is shown in Fig. 4, which gives average values for all 72 eyes. After the patient had spent I hour in complete darkness the changes in intraocular pressure were recorded over three 5-minute periods. In the first period during which the patient was still in darkness, there was a fall of $\mathrm{I} \cdot 8 \mathrm{~mm} . \mathrm{Hg}$; in the second period, during which the room was fully illuminated, there was a fall of $3.8 \mathrm{~mm}$. $\mathrm{Hg}$; in the final 5 -minute period, in which the room lights were kept on, there was further fall of only $0 \cdot 4 \mathrm{~mm}$. $\mathrm{Hg}$. Thus, the greatest fall in pressure occurred soon after the room lights were switched on, and this was so regardless of whether all eyes were considered together as in Fig. 4 or whether they were subdivided into glaucomatous and non-glaucomatous or treated and untreated groups. It is also to be noted that the final intraocular pressure was, on the average, $\mathrm{I} \cdot 3 \mathrm{~mm}$. $\mathrm{Hg}$ below the pressure recorded before the patient was put in the dark room, and there was a very high correlation between the rise in intraocular pressure in darkness and the total fall in pressure afterwards. There was also a significant correlation between the rise in pressure and the fall which took place in the first 5 minutes after switching the room lights on $(t=0 \cdot 48,0 \cdot 0$ I $>\mathrm{P}>0)$.

Fig. 4 shows that the intraocular pressure was determined on five occasions in the course of the test, and that on each occasion three tonometric readings were taken. In connection
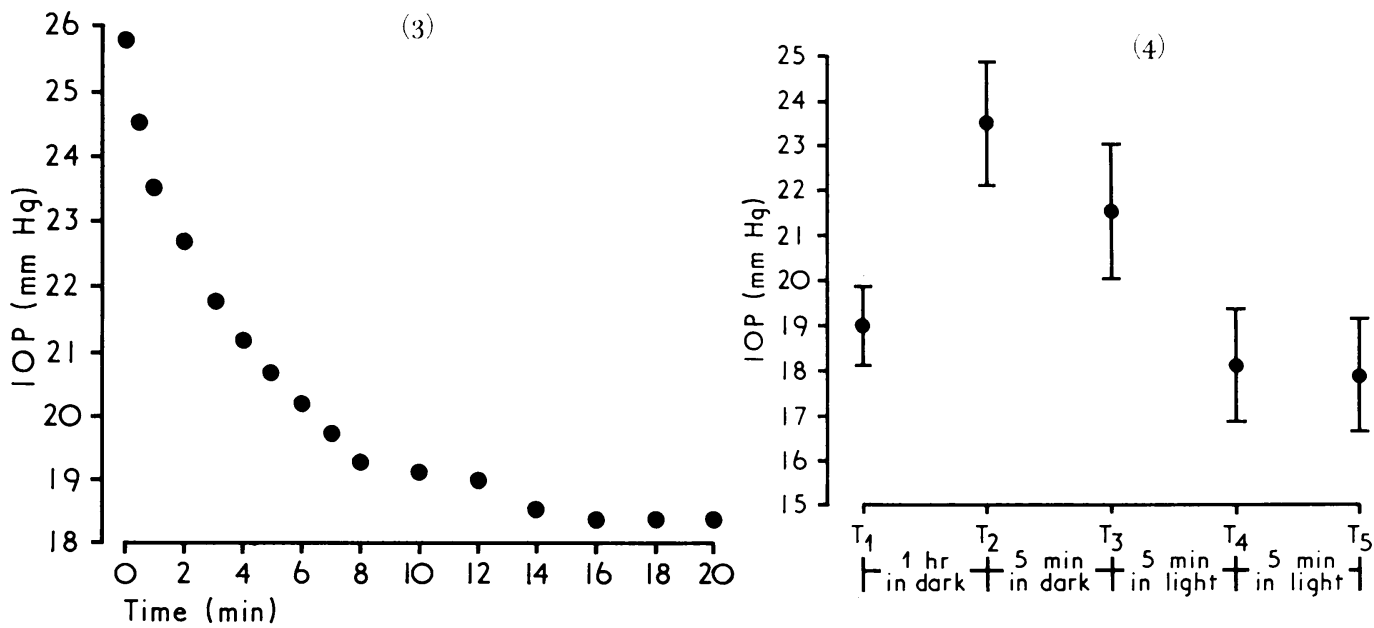

FIG. 3 Fall in intraocular pressure after the dark-room test. First procedure: the lights were switched on immediately after the tension had been taken after I hour in darkness

FIG. 4 Fall in intraocular pressure after the dark-room test. Second procedure: Tension $T_{2}$ was taken immediately after $\mathbf{I}$ hour in darkness but the lights were not switched on until Tension $T_{3}$ had been taken 5 minutes later; Tensions $T_{\Delta}$ and $T_{5}$ were taken in the light

Solid circle shows mean value of intraocular pressure; vertical line represents two standard errors above and below the mean. 
Table IV Successive tonometric readings taken immediately after I hour in darkness

\begin{tabular}{|c|c|c|}
\hline $\begin{array}{l}\text { Tonometric } \\
\text { reading }\end{array}$ & First & Third \\
\hline Mean $(\mathrm{mm} . \mathrm{Hg})$ & $25 \cdot 36$ & $25 \cdot 13$ \\
\hline \multirow[t]{2}{*}{ No. of eyes } & 72 & 72 \\
\hline & $\begin{array}{l}t=0.5 \\
\mathrm{P}>0.5\end{array}$ & \\
\hline
\end{tabular}

with the rate of fall in pressure after the dark-room test, it was important to discover if the third of these three successive tonometric readings was lower than the first, particularly in the case of the readings taken immediately after the patient had been in darkness for I hour; Table IV summarizes these results.

\section{Discussion}

Under the conditions of these experiments, the whole of the rise in intraocular pressure in the dark-room test could have been an effect of darkness, or part of it could have been due to other factors such as $(a)$ the effect of the local anaesthetic drops, $(b)$ spontaneous variation in the intraocular pressure during the period of the test, or $(c)$ an effect of air-tight occlusion of the eyes by the goggles worn in the dark room. A comparison of the rises in pressure with the spontaneous variations in a comparable series of eyes (Fig. I) rules out any overall contribution from factors $(a)$ and $(b)$, while the experiments done with goggles in the light eliminated factor $(c)$.

It is clear, therefore, that the rise in intraocular pressure recorded in these experiments is to be regarded mainly as an effect of darkness, and, when it comes to a consideration of how much this is brought about by closure of the angle of the anterior chamber, the results are in general agreement with those of earlier workers (e.g. Higgitt, 1954; Foulds, 1957), who found that rises in pressure of several $\mathrm{mm}$. $\mathrm{Hg}$ occurred in eyes which could be regarded as having no predisposition to closure of the angle. The contribution of the latter mechanism to the rise in pressure in some eyes has been thoroughly substantiated, however, by gonioscopic observation (Ross, 1953; Higgitt, 1954) and by tonography (Foulds, 1956). In the present study, evidence that angle closure was partly responsible for the rise in pressure in some eyes was provided by the finding that the average rise in 44 eyes with narrow angles was $5.50 \mathrm{~mm}$. $\mathrm{Hg}$, and that in 43 eyes with medium or wide angles was $3.81 \mathrm{~mm}$. $\mathrm{Hg}$, this difference being significant. Evidence that some factor other than angle closure was involved was provided by the substantial rise in pressure found in eyes with medium and wide angles, in eyes on miotics, and in eyes with peripheral iridectomies. The rise in pressure associated with this other factor showed a significant positive correlation with the initial pressure in the eye. This correlation can be seen in Table II to be highly significant for eyes with medium, medium-wide, and wide angles, and significant for eyes with mediumnarrow angles; it is not significant, however, for eyes with narrow and medium-narrow angles, presumably because of the additional factor of angle closure which is not dependent upon the initial pressure in the eye.

The general lack of effect of miotics (usually pilocarpine or eserine) upon the response of the pressure in eyes with narrow angles is surprising but, as can be seen in Table III, this result is quite clear. It means, presumably, that the factor which is not associated with 
angle closure played a frequent and substantial part in bringing about the rise in pressure in these eyes.

The first procedure showed that, when the room lights were put on again at the end of the dark-room test, the fall in intraocular pressure followed an exponential course in time; reference to Fig. 3 shows that, on the average, there was a fall of about $5 \mathrm{~mm}$. $\mathrm{Hg}$ in the first 5 minutes after the test. In the second procedure, the patient was exposed to much less light in the first five minutes after the test; only a small amount of light from a dimmed torch used when instilling the local anaesthetic and fluorescein drops, and the blue light of the applanation tonometer, have to be taken into consideration. The average fall in pressure in the first 5 minutes after the test in the second procedure was only $\mathrm{I} \cdot 8 \mathrm{~mm}$. $\mathrm{Hg}$ and it would seem that the different amounts of light reaching the eye account for the different rates of fall in pressure in the first as compared with the second procedure.

From the strictly practical point of view, it is important to know whether the light which necessarily falls upon the eye during instillation of the drops and during applanation tonometry reduces the intraocular pressure by a significant amount and so invalidates the result of a dark-room test conducted in this way. In the second procedure, there was an average fall of $\mathrm{I} \cdot 8 \mathrm{~mm}$. $\mathrm{Hg}$ in the first five 5 minutes (still in darkness) immediately after the dark-room test. Also, Table IV shows that there was an average fall of $0.23 \mathrm{~mm} . \mathrm{Hg}$ between the first and third tonometric readings taken immediately after $\mathrm{I}$ hour in darkness; the three readings were taken over a period of about i 5 seconds and this would suggest a rate of fall in pressure of $\mathrm{I} \mathrm{mm}$. $\mathrm{Hg}$ per minute. If this rate had persisted, the expected fall in pressure in 5 minutes would have been about $5 \mathrm{~mm} . \mathrm{Hg}$ (as it was in the first procedure), whereas the observed fall in the second procedure was only $\mathrm{I} \cdot 8 \mathrm{~mm}$. $\mathrm{Hg}$. From this one may conclude that, as soon as the light needed for tonometry was removed, the intraocular pressure fell at a rate of less than $\mathrm{I} \mathrm{mm}$. $\mathrm{Hg}$ per minute. It was necessary to use only a very small amount of light to instil drops in preparation for tonometry, and it is therefore unlikely that the pressure fell significantly during this period; furthermore we have seen that, during the period in which three tonometric readings were taken, there was a fall of only $0.23 \mathrm{~mm}$. Hg (Table IV) and also that there was no significant difference between the rise in pressure in right eyes and that in the left, the right always being measured before the left. Altogether, one may conclude that almost the entire rise in pressure during the darkroom test can be recorded by applanation tonometry, provided that attention is paid to details of procedure as mentioned above.

As regards the fall in intraocular pressure after the dark-room test, one could postulate that this is spontaneous or is due to repeated tonometry or to the repeated instillation of local anaesthetic drops or that it is the result of some action of light upon the eye, causing directly or indirectly (e.g. through the cardiovascular system) a fall in intraocular pressure. The fact that there was a fall in pressure in every eye rules out the possibility of a spontaneous variation. Reference to Fig. 4 shows that the fall in intraocular pressure was fastest in the second 5-minute period after darkness in the second procedure, and this means that, although repeated tonometry and repeated use of local anaesthetic drops may have some effect, it is the exposure of the eye to light which is the most important factor in reducing the pressure. The most obvious general explanation is that removal of light falling on the eye sets in motion a mechanism quite separate from angle closure, which raises intraocular pressure, while the subsequent exposure to light reverses this mechanism; the significant correlation of the rise in pressure with its subsequent fall supports this hypothesis.

With regard to the nature of this mechanism, several possibilities need to be examined. Of the three factors which are responsible for maintaining the level of intraocular pressure, 
viz. rate of aqueous production, resistance to aqueous outflow, and episcleral venous pressure, it is the second which is involved in the orthodox explanation of the response of the intraocular pressure to darkness. According to this explanation, darkness dilates the pupil, and in the eye with a narrow angle the outflow of aqueous humour is obstructed by iridocorneal contact; this explanation is acceptable, but it does not concern us at the moment because we have to explain the rise that occurs without closure of the angle, although it is possible that darkness could increase the resistance to aqueous outflow in some other way. The component that concerns us has been regarded in the past as "neurovascular", a term \& which can be taken to include, for example, general or local mechanisms which alter $\vec{\circ}$ episcleral venous pressure or aqueous production, and so caus : a persistent change of $\overrightarrow{\vec{H}}$ elevation in intraocular pressure. Another possibility, suggested by Feigenbaum (I928, I930), is that darkness causes dilatation of the choroidal bloodvessels; even if there were some $\frac{0}{0}$ hitherto unknown mechanism by which this occurs, it is st ll necessary to remember that, ơ if such a vasodilatation produced only a volumetric change in the eye, there would be a $\dot{\omega}$ temporary rather than a persistent rise in pressure. In fact, little is known of the course in time of the rise in intraocular pressure in darkness and this, together with pharmacological studies, would seem a useful line to follow in continuing these investigations.

The problem has not only theoretical but also practical interest. In the management of glaucoma, it is sometimes helpful to be able to assess the severity of the threat of angle closure. In some eyes, the dark-room test may provide unequivocal proof of a dangerous situation, but when the response of the intraocular pressure is less marked it would be useful to know how much was due to angle closure and how much to another factor.

\section{Summary}

(I) The Goldmann applanation tonometer can be used for the dark-room test, provided that certain conditions are observed. The results of this investigation proved that the amount of light needed for tonometry is too small to cause a significant error in the measurement of the rise in pressure in the eye.

(2) There are at least two factors involved in the elevation in pressure in darkness. One factor is related to narrowness of the angle, the other is not.

(3) The average rise in pressure is greater in eyes with narrow angles than in those with medium and wide angles.

(4) Significant rises in pressure occur in some eyes on miotics or after glaucoma surgery.

(5) There is a positive correlation between the rise in pressure and the initial pressure in eyes with medium and wide angles but not in eyes with narrow angles.

(6) In the fall in pressure after the dark-room test, exposure to light is the most important factor. The final pressure reached is usually lower than the initial pressure before the test.

\section{References}

feigenbaum, A. (1928) Klin. Mbl. Augenheilk., 80, 577

(1930) "Acta XIII int. Congr. Ophthal., Amsterdam, 1929", vul. 2, p. 49 I

FENTON, P. J. (1965) Brit. J. Ophthal., 49, 205; 504

Foulds, w. s. (1956) Trans. ophthal. Soc. U.K., 76, 83

(1957) Brit. 7. Ophthal., 4x, 200

GLOSTER, J. (I966) "Tonometry and Tonography". Churchill, London

HigGTT, A. C. (1954) Brit. 7. Ophthal., 38, 242

LEYDHEGKer, W. (1960) “Glaukom. Ein Handbuch". Springer, Berlin

Ross, м. G. (1953) Amer. 7. Ophthal., 36, 640 